\title{
EuroSpine Task Force on Research: support for spine researchers
}

\author{
Margareta Nordin $^{1} \cdot$ Federico Balagué $^{2} \cdot$ Kim Burton $^{3} \cdot$ Christine Cedraschi $^{4}$. \\ Pierre Côté $^{5} \cdot$ Björn Rydevik $^{6} \cdot$ L. Rachid Salmi ${ }^{7,8,9} \cdot$ Marek Szpalski $^{10}$
}

Published online: 20 October 2015

(C) Springer-Verlag Berlin Heidelberg 2015

\section{Introduction}

In recognition of the value of research to the practice of spine care, the President and Secretary (Federico Balagué and Ferran Pellisé) of EuroSpine, asked Margareta Nordin to set up a Task Force Research (TFR) for EuroSpine during summer 2011. The concept was to stimulate, facilitate and promote a research community within the society, through two main functions: (1) distribution of EuroSpine funds to researchers; (2) develop and deliver research training/education courses. What has the EuroSpine TFR accomplished since its inception?

\section{The creation of the TFR}

To reflect and meet the demands of the society around research, the TFR is necessarily a multidisciplinary group. Certain criteria for participating in the taskforce were

Margareta Nordin

margareta.nordin@nyu.edu

1 Department of Orthopedic Surgery, New York University, New York, NY, USA

2 HFR Fribourg-hôpital cantonal, Fribourg and Faculty of Medicine, University of Geneva, Geneva, Swizterland

3 Centre for Applied Psychological and Health Research, University of Huddersfield, Huddersfield, UK

4 Division of Clinical Pharmacology and Toxicology, Multidisciplinary Pain Centre \& Division of General Medical Rehabilitation, Geneva University Hospitals and University of Geneva, Geneva, Switzerland

5 Canada Research Chair, in Disability Prevention and Rehabilitation and Faculty of Health, Sciences, University of Ontario Institute of Technology (UOIT), Toronto, ON, Canada deemed important. A member of the TFR must have a solid research background, published at least 50 articles in peerreviewed journals, must have a track record of funding from national and/or international funding bodies and must have a track record of reviewing grants and promoting research. The TFR had to represent Europe but highly qualified researchers outside Europe were also invited. Members of the taskforce are volunteers, appointed for a 3 -year term, with a possibility to be reappointed. There are currently eight members in the TFR including the chair.

\section{TFR tasks and representation within EuroSpine}

The EuroSpine Executive Committee has been very clear in their directives to "give back" to the members, so a primary task for the TFR was to set up rules for the distribution of funding for EuroSpine researchers, along with

6 Department of Orthopaedics, University of Gothenburg, Sahlgrenska University Hospital, Gothenburg, Sweden

7 Univ. Bordeaux, ISPED, Centre INSERM U897Epidemiologie-Biostatistique, 33000 Bordeaux, France

8 INSERM, ISPED, Centre INSERM U897-EpidemiologieBiostatistique, 33000 Bordeaux, France

9 CHU de Bordeaux, Pole de sante publique, Service d'information medicale, 33000 Bordeaux, France

10 Department of Orthopedic and Trauma Surgery, Iris South Hospitals, Université Libre de Bruxelles, Brussels, Belgium 


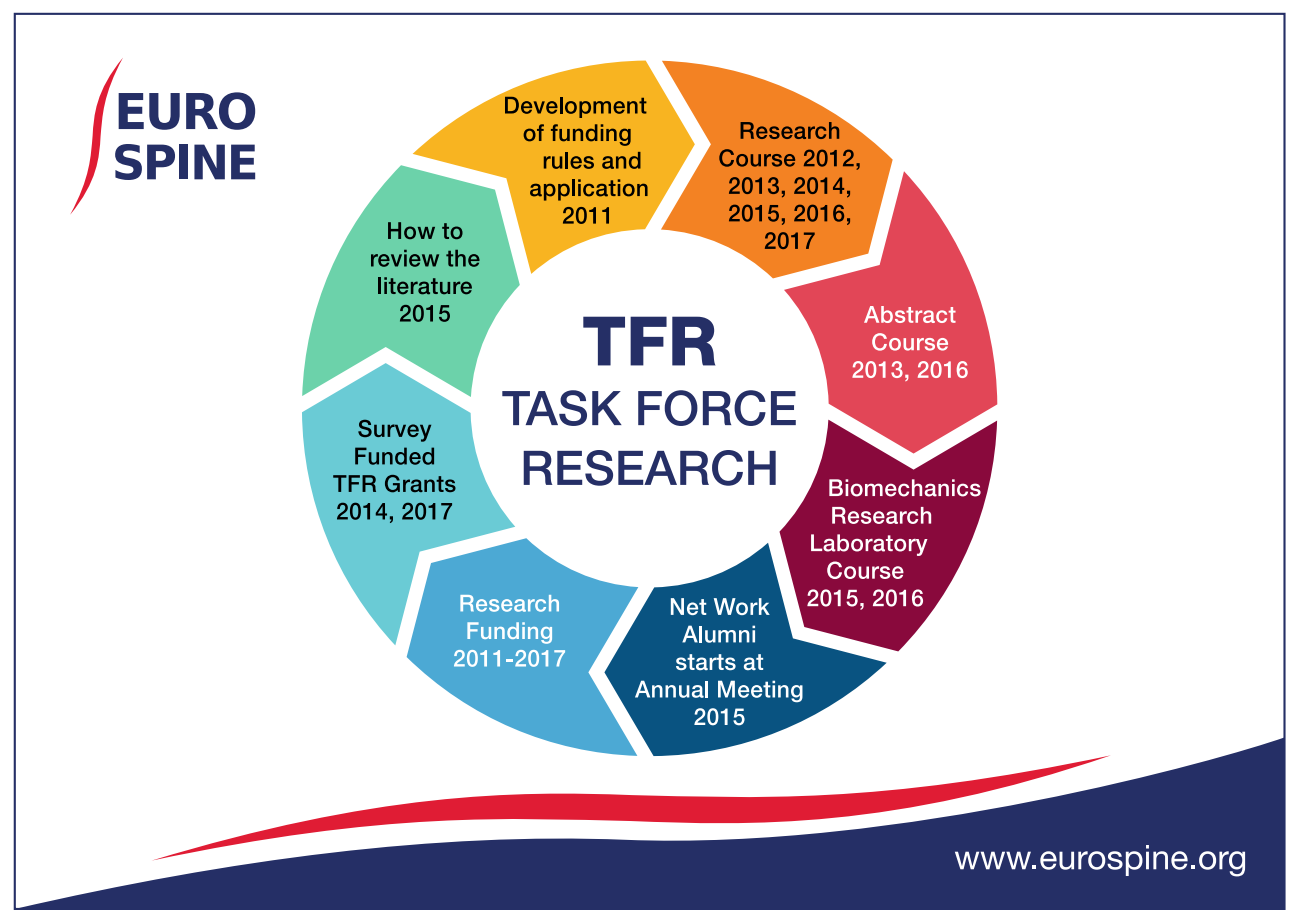

Fig. 1 Task force on research (TFR) past and planned activities 2011-2017

a mechanism for judging applications. The first funds were distributed in 2011.

The other primary tasks for the TFR were to develop and deliver a research training course, designed as a "crash course" in clinical research design for busy clinicians. TFR has now had 4 courses, one each year with about 20 participants per year.

TFR has continued to develop its remit, taking on additional tasks. Figure 1 shows the accomplishments from 2011 to June 2015, along with planned activities up to 2017.

The chair and/or members of TFR participate actively in the EuroSpine annual and spring meetings, and the TFR chair represents the research council in the EuroSpine Executive Committee. Thus, TFR is well integrated in the EuroSpine decision process.

\section{What will the future bring?}

The expansion of TFR activities has the support of the EuroSpine Executive Committee, with the main objectives of the TFR remaining to support research, to educate researchers, and to foster an active research community within the society.

\section{EuroSpine TFR funding for grant applications}

Before TFR could begin to allocate funds to members of EuroSpine, we had first to set up a funding system that was competitive, yet transparent and free from conflicts of interest. TFR reviewed several national and international funding organizations to set up a trustworthy system of grant review, select the categories for the type of grants, and develop suitable communication with applicants. We reviewed the procedures used by US, Canadian, French, Swedish, UK and Swiss funding organizations and took the optimal elements from these funding bodies, and created a grant funding mechanism for EuroSpine members. The system is paperless, being accessed on EuroSpine website under the tab research (http://www. eurospine.org/the-sse-funding-for-research.htm). All the steps were approved by the EuroSpine Executive Committee. The available research funds each year are allocated by the Executive Committee and ratified by the EuroSpine general assembly. In 2011, TFR could start to distribute funding.

The TFR has three grant categories as of 2015: (1) Development Grant-up to 10,000 Euros, (2) Pilot Study Grant for 1 year-up to 30,000 Euros and (3) Main Grant over 3 years-up to 100,000 Euros (http://www.eurospine. org/p31001608.html). The idea behind the three types of grant was that a research group could ask for funding to develop a research question, then apply for funding for a pilot study to test the research question. The TFR had reviewed other funding organizations and only few organizations fund development grants and/or pilot studies, which we considered especially important for spine researchers. If the pilot testing is successful, the research 
group may then apply for a larger grant through other larger funding agencies. The main purpose of TFR is not to fund large-scale studies because funds are limited. However, it was recognized that members would have important research questions that required moderate-scale funding extending over a few years, so this third category was incorporated.

Each eligible application is initially evaluated by two TFR members (who have to sign a no-conflict form), and then discussed by the whole team at an annual meeting. External reviewers can be contacted if the TFR deems not to have the expertise for the grant content. Applications are evaluated against TFR criteria covering novelty, scientific robustness, expertise, environment, etc. Scoring is on a scale from 1 to 9 (1 being the highest quality, and 9 the least fundable). The cut off for funding has been around 3-4. The turnaround time for funding is less than 5 months, which is key for eager spine researchers with a novel research question. The type of studies and grant amount funded until now can be viewed on the website (http://www.eurospine.org/ p31001607.html). One-third of the studies funded have been pilot studies, but cohorts, randomized control trials and other studies including basic science, systematic review, reliability and qualitative studies have also received funding from TFR.

TFR has distributed back to EuroSpine members a total of 548,573 Euros over 2011-2015.

\section{EuroSpine TFR research courses}

The research education focuses on clinical research design, with specialized research courses such as biomechanical laboratory testing and, perhaps, a basic science research design and a best laboratory practices course in the future. These courses are 4-5 days and receive CME accreditation and are partially funded by EuroSpine to create a skilled research community. The TFR has presented four clinical research design courses in different parts of Europe (Istanbul, Prague, Dublin, Barcelona) and the next clinical research course is in Milan on March 7-11, 2016. The Biomechanics Laboratory research course will be a novel joint venture between the University of Ulm and EuroSpine. Basic science research courses are currently being discussed by the TFR.

The course feedback has been very favorable. The course involves hard work from participants with half-day didactic lectures, half-day group work to create a research protocol and, perhaps most importantly, questions and answers in small study groups with the faculty. In all we attracted 77 participants over 4 years for the Clinical Research Design course.
The research courses are also meant to introduce the participants to EuroSpine TFR funding for research. This was a natural development by the TFR, to foster excellence in research design linked to potential funding possibilities. Participants in the research course would then be better prepared to apply for a EuroSpine TFR grant. Several of the research course participants have applied for funding, which is a success of system even if the application was not funded. The TFR is creating a research community that is active.

\section{Evaluation of TFR grant system for funding 2014}

In 2014, an independent survey was made of the TFR grant recipients 2011-2013. All 15 funded applicants were approached, with $100 \%$ response rate. The evaluation was indeed very positive. TFR had at the time funded projects in ten countries, with about $40 \%$ having women as principal investigators. At the time of the survey 11 abstracts had been accepted by the International Society for the Study of the Lumbar Spine, EuroSpine, Spine Week, Scoliosis Research Society, and National Spine Societies (UK, CH). Several of the EuroSpine TFR grant recipients had received larger funding from outside agencies after a pilot study (e.g., AOSpine, Global Stem Cell Technologies, Mäxi Stiftung) or supplemental funding from EuroSpine. Three articles had been accepted in European Spine Journal, British Medical Journal, and Spine, and an additional six articles were submitted in 2014 to peer-reviewed journals.

Some final comments of the recipients were: "It might be a good idea to put the details of past grantholders and links to their publications on the corresponding web pages of EuroSpine SSE TFR? Might encourage others" and "Thank you very much for supporting our work with the TFR grant. The support was invaluable, and the work carried out was a great success. It also allowed us to establish a great international working group, with whom we hope to continue our collaboration in the future".

\section{The immediate and longer term future}

TFR has created an alumni meeting for all past participants in the research courses, together with TFR grant recipients, so they can meet and mingle at the EuroSpine annual meetings. This gathering will be with the new members committee so new and current EuroSpine members can exchange ideas and experience.

The collaboration with the University of Ulm for the Biomechanics Laboratory Research course is also new for 
2015. This course is currently under the patronage of EuroSpine, but from 2016 it will be a collaborative course for biomechanical researchers between EuroSpine and the University of Ulm. In 2015, EuroSpine TFR in collaboration with Institut Franco Européen de Chiropraxie (IFEC) is starting a course called "How to best review a scientific article". This first course will be in French upon demand from IFEC and subsequent one-day courses will be organized by TFR in English.

The EuroSpine TFR hopes to be able to increase the amount of funding available for research grants for EuroSpine members. Currently, we distribute $150,000-180,000$ Euros per year, but the goal is to double that amount within 2 years, to be achieved through various initiatives that are currently being discussed.
The TFR may become a regular committee within the statutes of EuroSpine to perpetuate its existence and continuing growth. A professional society that gives back to its members is, we believe, a society of excellence. Fostering young researchers and new groups of research scientists who produce high quality evidence published in peer-reviewed journals is a commendable aim for our society and promises better care for the spine patient. EuroSpine TFR believes this is the way to go and grow.

Compliance with ethical standards

Conflict of interest None. 